\title{
Vitamin K2-induced cell growth inhibition via autophagy formation in cholangiocellular carcinoma cell lines
}

\author{
MASANOBU ENOMOTO ${ }^{1}$, AKIHIKO TSUCHIDA ${ }^{1}$, KEISUKE MIYAZAWA ${ }^{2}$, TOMOHISA YOKOYAMA ${ }^{2}$, \\ HIDEAKI KAWAKITA ${ }^{1}$, HIROMI TOKITA ${ }^{1}$, MUNEKAZU NAITO $^{3}$, MASAHIRO ITOH $^{3}$, \\ KAZUMA OHYASHIKI ${ }^{2}$ and TATSUYA AOKI ${ }^{1}$ \\ ${ }^{1}$ Third Department of Surgery, ${ }^{2}$ First Department of Internal Medicine and \\ ${ }^{3}$ Department of Anatomy, Tokyo Medical University, Tokyo, Japan
}

Received August 9, 2007; Accepted September 17, 2007

\begin{abstract}
Vitamin K2 (MK4) has antitumor effects on various types of cancer cell lines in vitro, and its efficacy has also been reported in clinical applications for patients with leukemia, myelodysplastic syndrome, and hepatocellular carcinoma (HCC). However, details of the mechanism of the antitumor effects of MK4 remain unclear. In the present study, we examined the antitumor effects of MK4 on cholangiocellular carcinoma (CCC) cell lines and its mechanism of action using the HL-60 leukemia cell line that exerts MK4induced cell growth inhibition via apoptosis induction and cell cycle arrest as a control. MK4 exerted dose-dependent antitumor effects on all three types of CCC cell lines. However, apoptosis occurred in a smaller percentage of cells and there was less cell cycle arrest compared with other cancer cell lines studied previously, which suggested slight MK4-induced cell growth inhibition via apoptosis induction and cell cycle arrest. On the contrary, histopathological fidings showed a large number of cells containing vacuoles in their cytoplasm, and electron microscopic findings showed a large number of cytoplasmic autophagosomes and autolysosomes. These findings suggested evidence of autophagy-related cell death. Fluorescence microscopy following acridine orange staining revealed an increase in the number of cytoplasmic acidic vesicular organelles characteristic of autophagy. Moreover, there were few cells forming autophagic vesicles in the control group, while the percentage of cells containing vacuoles in the MK4-treated group increased with the duration of culture. These results suggested that, unlike in leukemia, gastric cancer, HCC, and
\end{abstract}

Correspondence to: Dr Akihiko Tsuchida, Third Department of Surgery, Tokyo Medical University, 6-7-1 Nishi-Shinjuku, Shinjuku-ku, Tokyo 160-0023, Japan

E-mail: akibobo@hotmail.com

Key words: cholangiocellular carcinoma, vitamin K2, chemoprevention, autophagy other cancer cells, the antitumor effects of MK4 on CCC cells are induced via autophagy formation.

\section{Introduction}

Vitamin K2 (VK2) is a fat-soluble vitamin that plays a role in the synthesis of prothrombin in the liver and affects the clotting system. VK2 has been reported to exert in vitro antitumor effects on malignant cells of various types of cancers including acute myeloid leukemia (AML), lung cancer, gastric cancer, and hepatocellular carcinoma (HCC) (1-5). Clinical investigations have also reported on the efficacy of VK2 describing a decreased number of blastic cells associated with oral medication of VK2 in patients with post-myelodysplastic syndrome AML (6) and a decreased percentage of blastic cells, an increased number of mature neutrophils, and improvement in decreased red blood cells and platelets in patients with myelodysplastic syndrome (MDS) (7). Furthermore, in a randomized controlled study in HCC patients judged as in complete remission following various treatments who were divided into a VK2-treated group and non-treated group, the VK2-treated group had lower recurrence, with a 3 -year accumulated recurrent rate of $65.5 \%$, compared with a rate of $92.2 \%$ in the non-treated group (8). Although factors including cancer cell-induced apoptosis, cell cycle arrest, and induction of differentiation are believed to contribute to the mechanisms of these in vitro and in vivo antitumor effects of VK2 (1-4), details remain to be examined.

Homologues of vitamin $\mathrm{K}$ consist of natural-found vitamins K1 (phytonadione: VK1) and K2 (menaquinone: VK2), and chemically-synthesized vitamin K3 (menadione: VK3). In general, antitumor effects are greatest in VK3, followed by VK2, and VK1 is believed to have the smallest effects $(5,9)$. Although VK3 is considered to exert a large antitumor effect (10), its safety has not been documented because of no clinical use. VK2 is denoted by menaquinone-n (MKn) according to the number of isoprenoid radicals that make up its side chain, and 14 of these (MK1-MK14) are found in nature. MK4 (menatetrenone), the VK2 used in the present study, has a geranylgeraniol radical as its side chain, which is clinically used for treatment of diseases such as osteoporosis and hypoprothrombinemia. Since VK2 has already been used in 
many clinical applications with few side effects (11), it is expected to be promptly used for clinical applications in cancer treatment.

In recent years, attention has been drawn to autophagy, like apoptosis, as a programmed cell death (PCD) (12). Autophagy fundamentally refers to a process where cells enclose their own cell organelles with an isolated membrane and deliver them to lysosomes for degradation, serving as a nonspecific degradation mechanism of autologous proteins. Autophagy, when induced by various types of stresses such as starvation, forms and re-utilizes the amino acid pool. Autophagy is a biological phenomenon inherent in all eukaryotes essential for vital activities. For this reason, autophagy has been recognized as a cytoprotective mechanism for cells to protect themselves from extracellular stresses $(13,14)$. Furthermore, since autophagy-related gene knock-out mice were reported to exhibit an increased risk of developing cancers, autophagy has also been recognized as a tumor suppressor $(15,16)$. However, in a culture using cells in which autophagy-related genes were suppressed by siRNA and to which an autophagy inhibitor, 3-methyladenine (3MA), was added, cytotoxic effects of some anticancer agents on cancer cells were diminished. Based on this result, the concept of caspase-independent cell death via autophagy, that is autophagic cell death (type II PCD), has been receiving increased attention (16-18).

We previously reported that VK2 has antitumor effects on cell lines of leukemia (1), MDS (19), lung cancer (2,3), and gastric cancer (4) via apoptosis induction and cell cycle arrest and that Bcl-2 overexpressed HL-60 cell lines by bcl-2 gene transfection (HL-60-bcl-2) are resistant to VK2-inducing apoptosis, but show differentiation-inducing effects via the induction of G1 arrest (1). We also reported that VK2 shows cancer protective effects in an experiment using hamster models of biliary carcinogenesis in pancreaticobiliary maljunction (20). However, the detailed mechanism of cancer inhibition of VK2 remains to be defined. In the present study, we performed experiments using cholangiocellular carcinoma cell lines to examine to what extent autophagy contributes to VK2-induced antitumor effects and their mechanism of action.

\section{Materials and methods}

Cell lines and reagents. VK2 (menaquinone-4: MK4) was supplied by Eisai Co. Ltd. (Tokyo, Japan). Three types of cholangiocellular carcinoma cell lines, TFK-1 (21), MEC (22), and HuCC-T1 (23), were provided by the Institute of Development, Aging and Cancer, Tohoku University (Sendai, Japan). The HL-60 leukemia cell line that exerts cell growth inhibition via apoptosis induction and cell cycle arrest with the addition of VK2 were used as a control (1).

All cell lines were cultured in RPMI-1640 medium (Gibco, Grand Island, NY, USA) with 10\% FBS (Hyclone, Logan, UT, USA) in an atmosphere of $5 \% \mathrm{CO}_{2}$ air at $37^{\circ} \mathrm{C}$. Adherent cholangiocellular carcinoma cell lines were treated and separated using a $0.25 \%$ trypsin and $0.02 \%$ EDTA solution during the logarithmic growth phase to form a cell suspension with an adjusted cell count of $5 \times 10^{4}$ cells $/ \mathrm{ml}$ for the following experiments.
Assessment of the cell growth inhibition in response to MK4. MK4 was added to each cell suspension $\left(5 \times 10^{4}\right.$ cells $\left./ \mathrm{ml}\right)$ to obtain final concentrations of $0.1,0.5,1,2,5,10,20,50$, and $100 \mu \mathrm{M}$. The cell suspensions were placed on each 96-well tissue culture plate (BD Biosciences, Bedford, MA, USA) to make five samples of wells containing $100 \mu 1$ of cell suspension for each concentration including the control group, and incubated for $96 \mathrm{~h}$. After adding a $10 \mu \mathrm{l}$ of reagent $(5 \mathrm{mM}$ WST-1, 20 mM HEPES, 1-methoxy PMS $5 \mathrm{ml}$ ) prepared using a cell counting kit (Dojindo Laboratories, Kumamoto, Japan) into each well, the optical density of each well was measured with a microplate reader equipped with a 450-nm filter to calculate the percentage of optical density for each concentration to the controls as the relative cell number.

Assessment of apoptosis induction. Cells undergoing apoptosis were assessed by flow cytometry using APO2.7 (clone 2.7 A6A3) mouse monoclonal antibody (mAb) (Immunotech, Marseille, France) that reacts to the 7A6 antigen expressed by cells undergoing apoptosis (19). Cultured cells were separated using a $0.25 \%$ trypsin and $0.02 \%$ EDTA solution and washed twice with PBS containing 5\% FBS. Then, $1 \times 10^{5}$ cells were placed in $0.5 \mathrm{ml}$ of PBS containing 5\% FBS and incubated with the addition of APO2.7 mAb and FITC-conjugated goat anti-mouse IgG mAb (Immunotech) for $30 \mathrm{~min}$ at $4^{\circ} \mathrm{C}$. Immunofluorescence was analyzed by flow cytometry using an Epics XL2 flow cytometer (Coulter Japan, Tokyo, Japan) to compare the percentage of APO2.7-positive cells in each cell line between the MK4-treated group and the control group.

Cell cycle analysis. For each cell line, two groups, with and without the addition of $50 \mu \mathrm{M}$ of MK4, were established and the cells were separated using a $0.25 \%$ trypsin and $0.02 \%$ EDTA solution after $96-\mathrm{h}$ incubation. The cell suspensions were stained with a solution containing $1 \%$ propidium iodide (Sigma), $100 \mu \mathrm{g} / \mathrm{ml}$ digitonin, $0.01 \% \mathrm{NaN}_{3}, 200 \mu \mathrm{g} / \mathrm{ml}$ RNase (Sigma), and $2.5 \% \mathrm{FBS}$ for $10 \mathrm{~min}$ at room temperature, and fluorescence intensity of the cells were measured using a flow cytometer (Epics XL2 flow cytometer). Based on this result, the populations of the $\mathrm{G} 0 / \mathrm{G} 1, \mathrm{~S}$, and $\mathrm{G} 2 / \mathrm{M}$ phases were analyzed using a cell-cycle analysis program, MultiCycle AV (Phoenix Flow Systems, San Diego, CA, USA) (1).

Immunoblotting for detection of Bcl-2. After each of the TFK-1 cell lines and the HL-60 cell lines was lysed using a lysis buffer solution (10 mM Tris- $\mathrm{HCl} \mathrm{pH} 7.8,150 \mathrm{mM}$ $\mathrm{NaCl}, 1 \% \mathrm{NP}-40,1 \mathrm{mM}$ EDTA, $10 \%$ glycerol, $1 \mathrm{mM}$ phanylmethylsulfonyl fluoride, $0.15 \mathrm{U} / \mathrm{ml}$ aprotinin, $10 \mathrm{mg} /$ $\mathrm{ml}$ leupeptin, $100 \mathrm{mM}$ sodium floride, $2 \mathrm{mM}$ sodium orthovanadate), determination of protein concentration was performed with a protein assay kit (Bio-Rad, Richmond, CA, USA). After the TFK-1 and the HL-60 cell lines at the same cell count were spinned down, the same amount of lysis buffer solution was added and solubilization of protein was performed under the same conditions. Then, the solubilized solutions with equalized protein content and cell count were fractionated on $15 \%$ SDS-PAGE and blotted to an Immobilon-P membrane (Bio-Rad). After reacting to antihuman Bcl-2 mAb (BD Biosciences Pharmingen, San Jose, 


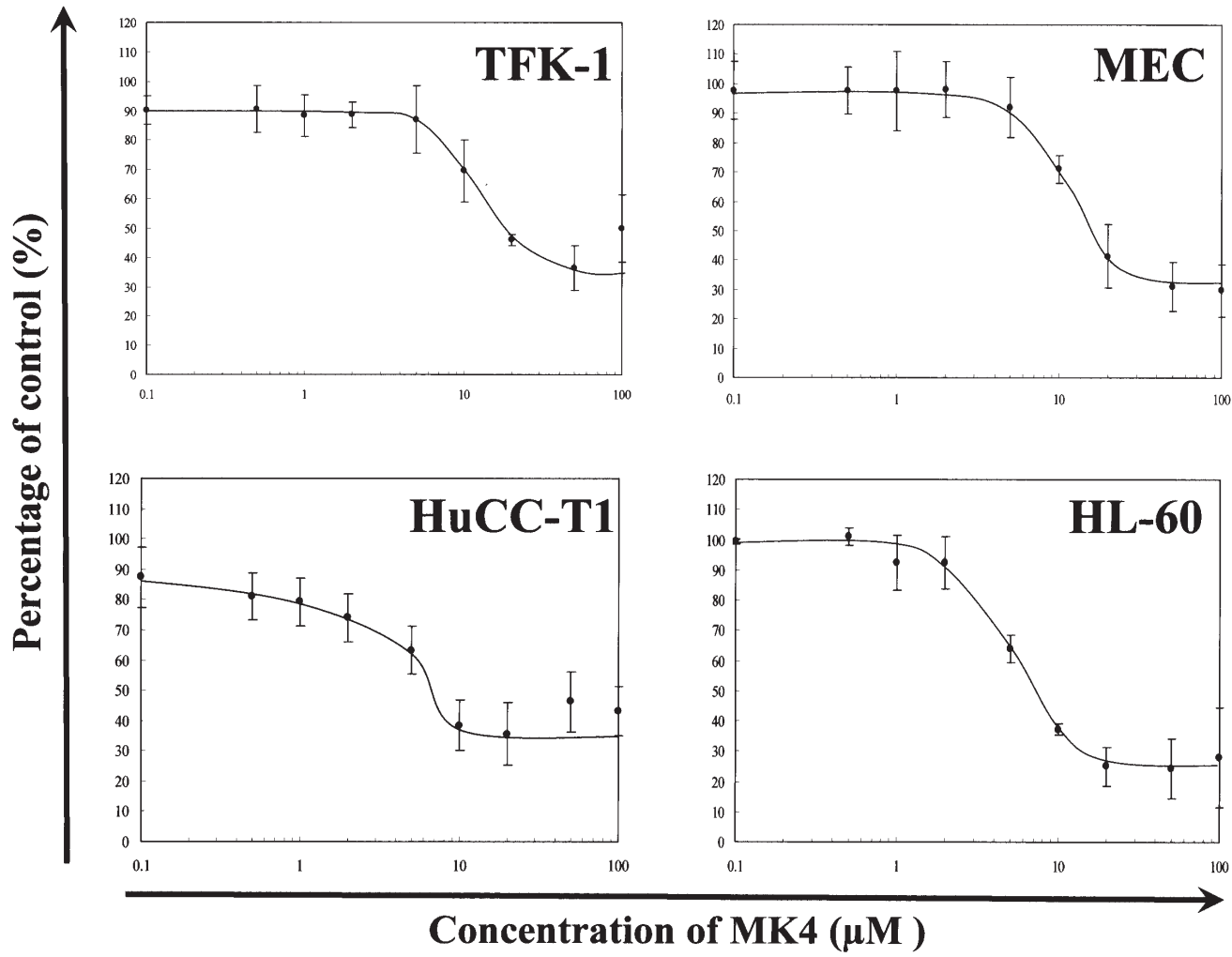

Figure 1. Cell growth inhibition of cholangiocellular carcinoma cell (CCC) lines after treatment with MK4. MK4 at the concentration range from 5 to $50 \mu \mathrm{M}$ exerted dose-dependent cell growth inhibitory effects on each of the three types of CCC cell lines. Dose-dependent cell growth inhibitory effects on HL-60 leukemia cell lines were observed at the concentration range of MK4 from 2 to $20 \mu \mathrm{M}$.

CA, USA), they were reacted to horseradish peroxidaseconjugated goat anti-mouse $\operatorname{IgG} \mathrm{mAb}$, and visualization of immunoreactive proteins was carried out using an ECL kit (Amersham Biosciences Co.).

Assessment of morphological changes. After adding MK4 and incubation, cell solutions were prepared. Specimens were prepared using cytospin 2 (Thermo Fisher Scientific Inc., Worcester, MA, USA) and the cell morphology was observed under May-Giemsa staining. Cells were classified into the following four types: 1) cells without significant morphological change, 2) polynuclear cells, 3) apoptotic cells, 4) autophagic cells, and were observed chronologically. Autophagic cells were defined as those with five or more vacuoles in the cytoplasm.

Electron microscopy. For TFK-1 cell line, two groups with and without the addition of $50 \mu \mathrm{M}$ of MK4 were established and the cells were fixed for $1 \mathrm{~h}$ using a $3 \%$ glutaraldehyde plus $2 \%$ paraformaldehyde in $0.1 \mathrm{M}$ cacodylate buffer $(\mathrm{pH} 7.3$ ) solution. Then, the cells were post-fixed using the same buffer solution to which $1 \% \mathrm{OsO}_{4}$ was added. After embedding in gelatin, the cells were observed by electron microscopy.

Detection of acid vesicular organelles (AVOs). Vital staining was performed using acridine orange (Polysciences, Warrington, PA, USA) to confirm the presence of acid vesicular organelles (AVOs), characteristic of autophagy. For TFK-1 cell line, two groups, with and without the addition of $50 \mu \mathrm{M}$ of MK4, were established and incubated for $96 \mathrm{~h}$, the cells were separated using a $0.25 \%$ trypsin and $0.02 \%$ EDTA solution. Acridine orange was dissolved in RPMI-1640 to reach a concentration of $1 \mu \mathrm{g} / \mathrm{ml}$. The cells were then cultured for $30 \mathrm{~min}$ in an atmosphere of $5 \% \mathrm{CO}_{2}$ air at $37^{\circ} \mathrm{C}$ and observed using a fluorescent microscope $(25,26)$.

Statistical analysis. The data are shown as means \pm SD. Comparisons between two groups were assessed using the Student's t-test. A p-value of $<0.05$ was considered to indicate a statistically significant difference.

\section{Results}

Growth inhibitory effects of MK4 in cholangiocellular carcinoma $(C C C)$ cell lines. MK4 at the concentration range from 5 to $50 \mu \mathrm{M}$ exerted dose-dependent cell growth inhibitory effects on each of the three types of CCC cell lines (Fig. 1). Observed 50\% inhibitory concentrations $\left(\mathrm{IC}_{50}\right)$ were 10.7 , 10.6, and $7.3 \mu \mathrm{M}$ for TFK-1, MEC, and HuCC-T1, respectively. In addition, dose-dependent cell growth inhibitory effects on HL-60 leukemia cell lines were observed at the concentration range of MK4 from 2 to $20 \mu \mathrm{M}$, with an $\mathrm{IC}_{50}$ of $6.8 \mu \mathrm{M}$.

Apoptosis induction. To confirm the effects of apoptosis on MK4-induced cell growth inhibition, the percentage of APO2.7 positive cells was measured by flow cytometry. In the cell lines TFK-1, MEC, and HuCC-T1, although the percentage of APO2.7 positive cells tended to slightly increase in the MK4-treated group, no statistically significant difference 


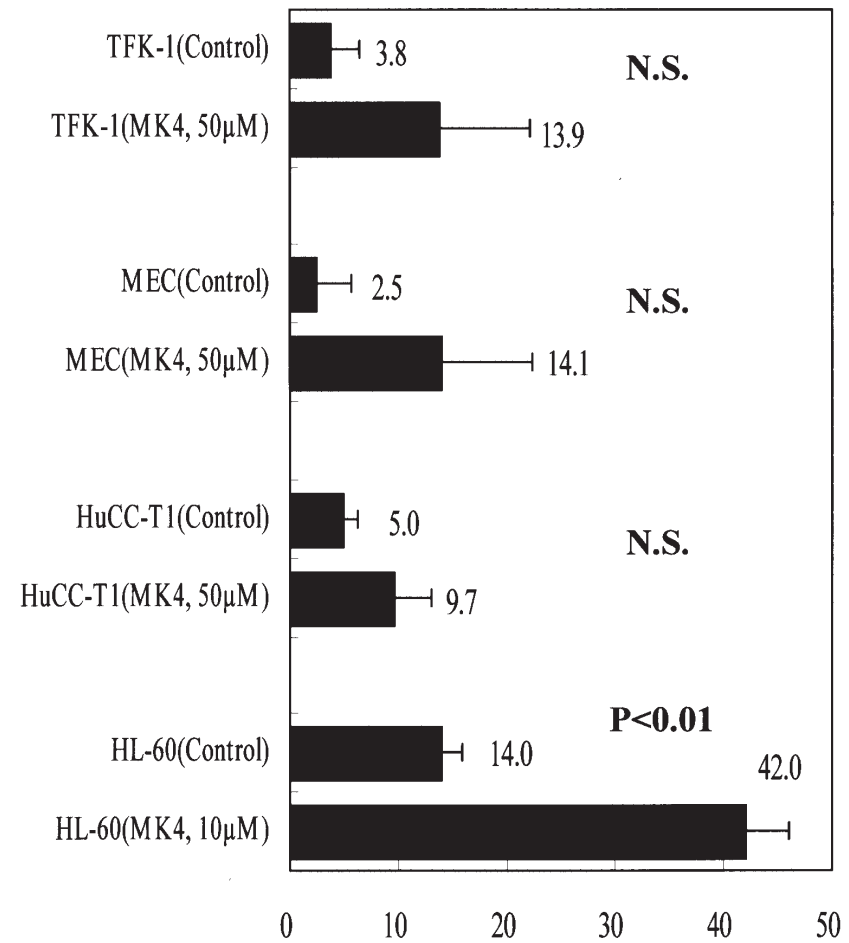

Percentage of APO2.7 positive cells (\%)
TFK-1
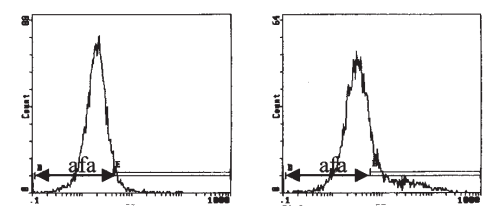

MEC
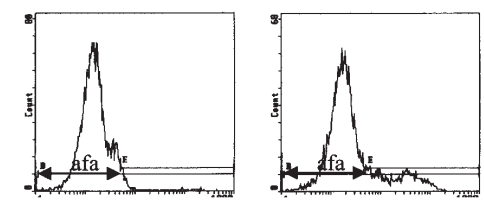

HuCC-T1

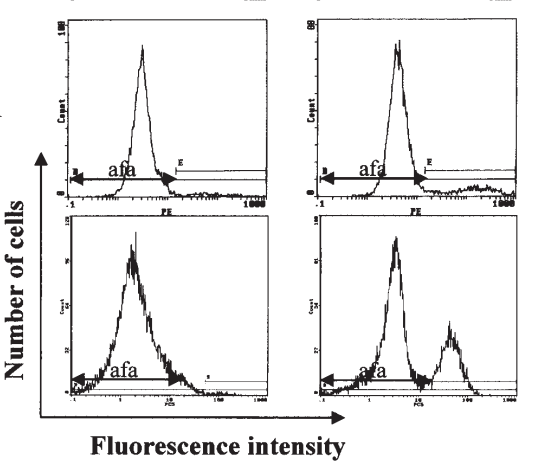

Control

MK4

APO2.7

Figure 2. Assessment of apoptosis induction in response to MK4 in cholangiocellular carcinoma cell lines. For all CCC cell lines, the percentage of APO2.7 positive cells tended to slightly increase in the MK4-treated group, while no statistically significant difference was observed as compared with the control group. For HL-60 cell lines, on the contrary, a statistically significant increase in the percentage of APO2.7 positive cells was observed in the MK4-treated group $(\mathrm{p}<0.01)$

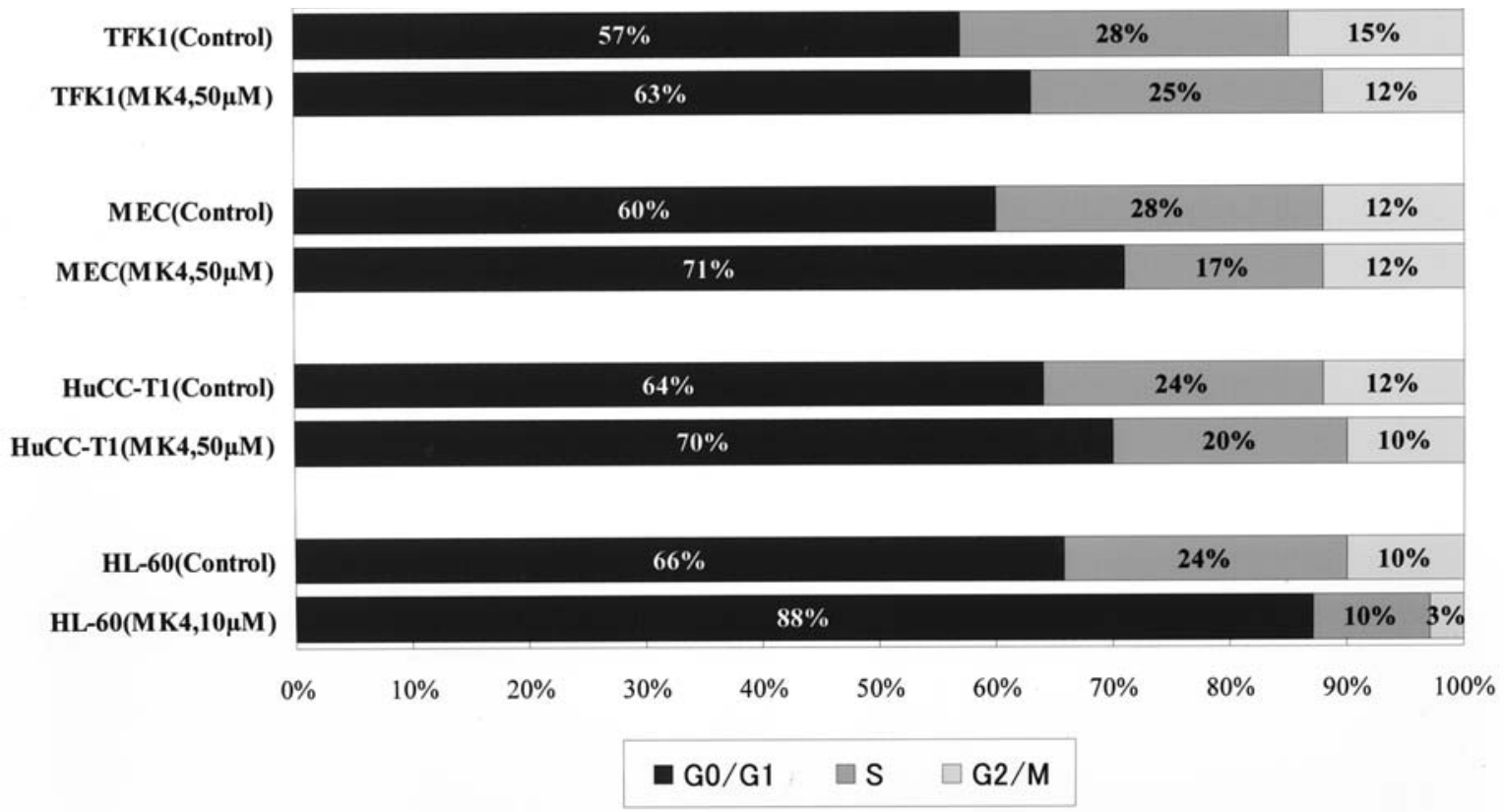

Figure 3. Cell cycle analysis after exposure to MK4 in cholangiocellular carcinoma cell lines. Among the cells that did not undergo cell death, the percentage of G0/G1-phase cells for HL-60 was $66 \%$ in the control group and $88 \%$ in the MK4-treated group, suggesting an obvious increase by MK4. On the other hand, the percentage of G0/G1-phase cells for TFK-1, MEC, and HuCC-T1 was 57, 60, and 64\% in the control group and 63, 71, and 70\% in the MK4-treated group, respectively. Although an increasing tendency was observed in each of the MK4-treated groups, the degree of cell cycle arrest was small.

was observed compared with the control group (Fig. 2). In the HL-60 cell lines, on the contrary, a statistically significant increase in the percentage of APO2.7 positive cells was observed in the MK4-treated group $(\mathrm{p}<0.01)$. Thus, MK4-induced cell growth inhibitory effects were observed in CCC cell lines; however, the degree of apoptosis induction 


\section{HL-60 TFK-1 HL-60 TFK-1}

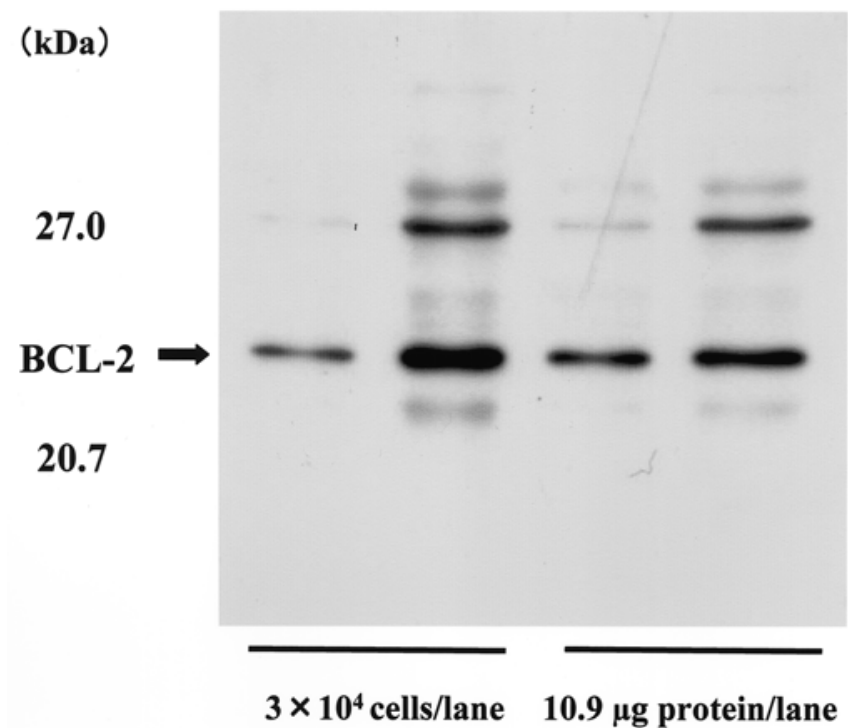

Figure 4. Comparison of Bcl-2 expression between TFK-1 and HL-60 cells. The expression level of Bcl-2 protein was compared between TFK-1 and HL-60 by immunoblotting with anti-Bcl-2 mAb. For standardization, two methods of measurement using equalized soluble protein content and cell counts were used. Both measurements showed a higher expression of Bcl-2 protein in TFK-1 than in HL-60.

was smaller than that observed in HL-60 leukemia cell lines. It was reported that overexpression of $\mathrm{Bcl}-2$ prevents apoptosis from being induced in HL-60, but G1 arrest of the cell cycle was induced by MK4 (1). Therefore, we considered that MK4-induced cell growth inhibition is attributable to the cytostatic action via cell cycle arrest.

Cell cycle analysis and expression level of Bcl-2. Among the cells that did not undergo cell death, the percentage of G0/G1phase cells for HL-60 was $66 \%$ in the control group and $88 \%$ in the MK4-treated group, suggesting an obvious increase by MK4. On the other hand, the percentage of G0/G1-phase cells for TFK-1, MEC, and HuCC-T1 was 57, 60, and 64\% in the control group and 63,71, and 70\% in the MK4-treated group, respectively. Although an increasing tendency was observed in each of the MK4-treated groups, the degree of cell cycle arrest was low (Fig. 3).

The expression level of Bcl-2 protein was compared between TFK-1 and HL-60. For standardization, two methods of measurement using equalized soluble protein content and cell counts were used. Both measurements showed a higher expression of Bcl-2 protein in TFK-1 than in HL-60 (Fig. 4).

Morphological appearance. Since we recognized that the effects of apoptosis and cell cycle arrest on MK4-induced cell growth inhibition were smaller than anticipated, we focused our attention on morphological differences. In HL-60 cells, the addition of MK4 resulted in typical apoptotic images including condensation of chromatin and nuclear fragmentation (apoptotic body). In TFK-1 cells, on the contrary, the addition of MK4 did not result in such apoptotic images, and a large number of cells containing vacuoles in their cytoplasms were observed, suggesting autophagyinduced cell death (12) (Fig. 5).

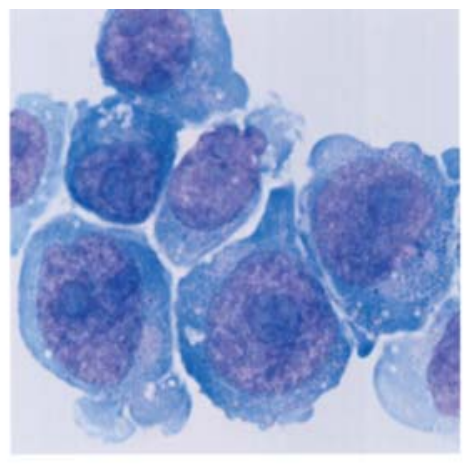

HL-60 (Control)

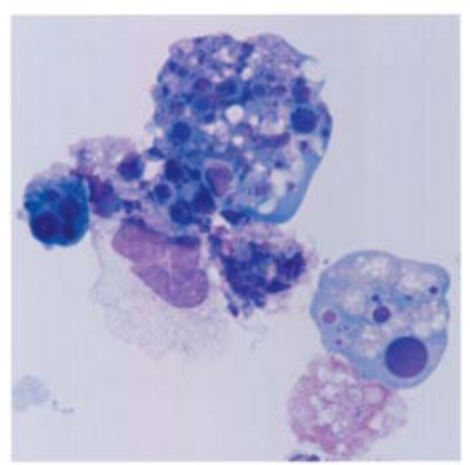

HL-60 (MK4, 10 $\mu \mathrm{M})$

Figure 5. Comparison of morphologic changes between TFK-1 and HL-60 cells after treatment with MK4. In HL-60 cells, the addition of MK4 resulted in typical apoptotic images including condensation of chromatin and fragmentation of nucleus. In TFK-1 cells, on the contrary, the addition of MK4 resulted in autophagy images including a large number of cells containing vacuoles in their cytoplasms. May-Giemsa staining: original magnification, $\mathrm{x} 1000$. 


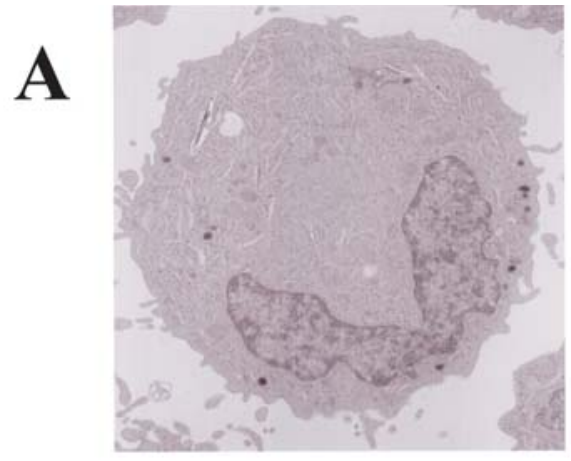

TFK-1 (Control)

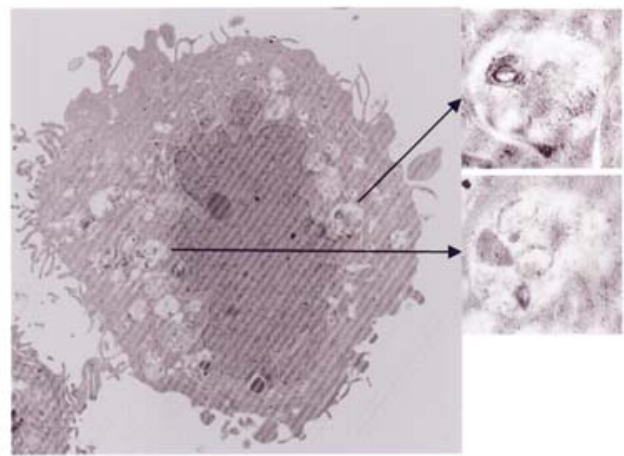

TFK-1 (MK4, 50 $\mu \mathrm{M})$

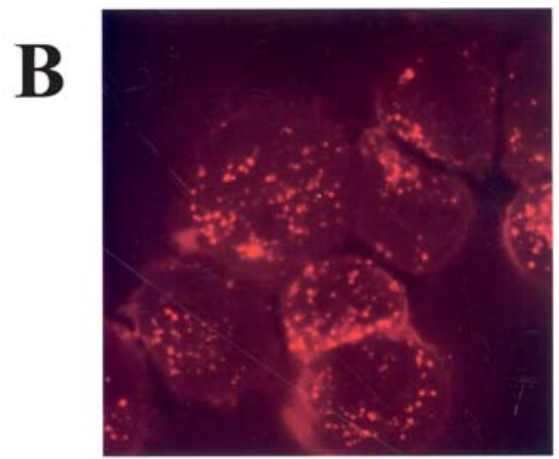

TFK-1 (Control)

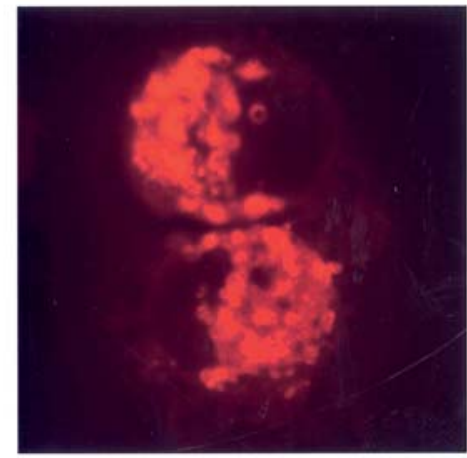

TFK-1 (MK4, 50 $\mu \mathrm{M})$

Figure 6. Induction of autophagy in response to MK4 in TFK-1 cells. (A) Electron microscopy showed a large number of cytoplasmic autophagosomes and autolysosomes in the MK4-treated group. (B) Fluorescence microscopy following acridine orange staining revealed an increase in the number of cytoplasmic acidic vesicular organelles (AVOs) characteristic of autophagy in the MK4-treated group.

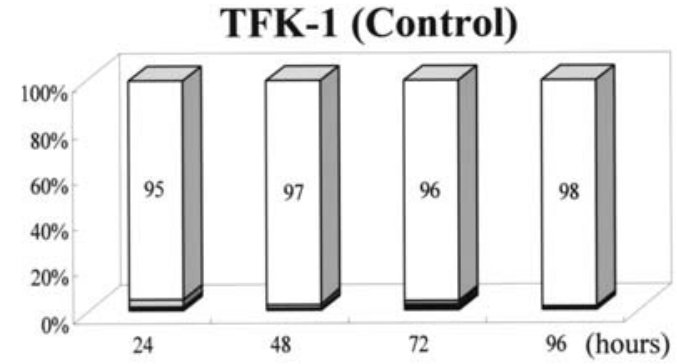

TFK-1 (MK4, 50 $\mathrm{MM})$

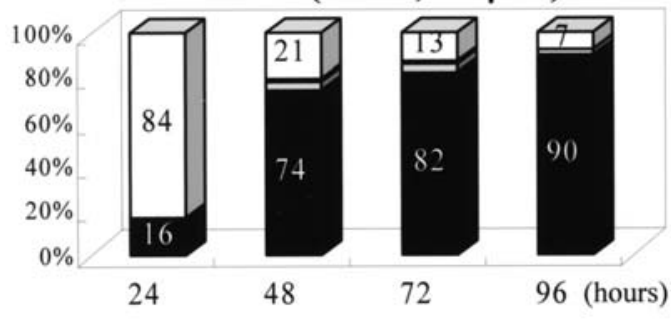

$\square$ Cells with no significant morphologic change $\square$ Polynuclear cells

$\square$ Apoptotic cells

autophagic cells (with vacuoles)

Figure 7. Chronological morphologic changes of TFK-1 cells during exposure to MK4. Morphological observation in the MK4-treated and control group showed that there were few cells forming vacuoles in the control group. In the MK4-treated group, on the contrary, the number of cells with vacuoles significantly increased at 48-h post culture. Moreover, the percentage of the cells increased with the duration of culture and cells with vacuolation accounted for $\sim 90 \%$ of the cells at $96 \mathrm{~h}$
Autophagy induction of TFK-1 cells in response to MK4. To confirm the evidence of autophagosome and autolysosome formation characteristic of autophagy, the following observation was performed. Electron microscopy showed a large amount of cytoplasmic autophagosomes and autolysosomes in the MK4-treated group (Fig. 6A). In the MK4treated group, fluorescence microscopy following acridine orange staining revealed an increase in the number of cytoplasmic acidic vesicular organelles (AVOs) characteristic of autophagy (Fig. 6B) (24-26). Moreover, chronological morphological observation in the MK4-treated and the control group showed that few cells formed vacuoles in the control group. In the MK4-treated group, on the contrary, the number of cells with vacuoles significantly increased at 48-h post culture. Moreover, the percentage of the cells increased with the duration of culture, and $\sim 90 \%$ of the cells showed vacuolation at $96 \mathrm{~h}$ (Fig. 7).

\section{Discussion}

We set out to determine whether VK2 has antitumor effects through the induction of autophagy on cholangiocellular carcinoma (CCC) cells. Although in vitro and in vivo antitumor effects of VK2 have been documented previously (1-8), details of the mechanisms of the effects remain unclear. Bouzahzah et al described that VK2 may increase the expression of jun and $c$-myc, leading to apoptosis induction and that it may also increase prothrombin and other VK2-dependent proteins, resulting in growth inhibition of hepatocellular carcinoma 
(5). In the study of the effects of VK2 using MDS-KZ cell lines derived from MDS patients, Nishimaki et al reported that the addition of VK2 led to decreased Bcl-2 expression and increased BAX expression, which caused depolarization of mitochondria membrane potential, release of cytochrome c, and activation of caspase- 3 , and thereby inducing cell death via apoptosis. They also reported that the addition of a caspase inhibitor, ZVAD, suppressed apoptosis induced by VK2. These results led to the speculation that mitochondria is a target organ for apoptosis by VK2 (27). Moreover, Miyazawa et al reported that examination of the effects of VK2 in an HL-60 leukemia cell line overexpressing Bcl-2 as a result of bcl-2 gene transfection (leukemia HL-60-bcl-2) showed the suppression of apoptosis in HL-60-bcl-2 cells due to the stabilization of mitochondrial membrane, while also showing an increase of G0/G1 phase and monocytic differentiation inducibility (1). These results suggest that the antitumor effects of VK2 are caused mainly by apoptosis induction and cell cycle arrest.

The present study demonstrates that the addition of VK2 resulted in dose-dependent cell growth inhibitory effects on all three CCC cell lines (Fig. 1). The $\mathrm{IC}_{50}$ for the three types of CCC cells ranged from 5 to $20 \mu \mathrm{M}$, which was slightly higher than the $\mathrm{IC}_{50}$ of 5 to $10 \mu \mathrm{M}$ for leukemia cell lines. When compared with the $\mathrm{IC}_{50}$ for the same type of solid tumor, gastric cancer and lung cancer cell lines, of 20 to 60 $\mu \mathrm{M}(4)$ and 10 to $50 \mu \mathrm{M}(2,3)$, respectively, the $\mathrm{IC}_{50}$ for CCC cell lines tended to be lower. In addition, previous studies reported that the $\mathrm{IC}_{50}$ for $\mathrm{HCC}$ cell lines ranged from 100 to $200 \mu \mathrm{M}$ and that certain $\mathrm{HCC}$ cell lines have only $<50 \%$ growth inhibition rate $(5,28)$. Based on these reports, it was considered that CCC cells were more sensitive to the antitumor effects of VK2 than hepatocellular carcinoma (HCC) cells.

In the present study, the apoptosis induction effect of VK2 on CCC cells was assessed using APO2.7. Although the percentage of APO2.7-positive cells tended to slightly increase (9-15\%) in each of the MK4-treated CCC cells, the apoptosis induction effect of VK2 in CCC cells was relatively small, compared with the rates of $\sim 50 \%$ for leukemia cell lines (1), 20 to $40 \%$ for gastric cancer cell lines (4), and $30-40 \%$ for lung cancer cell lines $(2,3)$. A possible reason for this smaller effect is that Bcl-2 expression is higher in CCC cells (TFK-1) than in HL-60 (Fig. 4). Regarding the cell cycle, although the percentage of G0/G1-phase cells tended to increase in the VK2-treated $(50 \mu \mathrm{M})$ group with a rate of $63.2 \%$ as compared with $57.4 \%$ for the TFK-1 control group, the percentage of G0/G1-phase cells was lower than that in HL-60 (Fig. 3). Furthermore, in a previous study from our group among four types of gastric cancer cell lines, all had $>70 \%$ G0/G1-phase cells and two had $\geq 80 \%$ G0/G1-phase cells (4). Although the in vitro antitumor effects of VK2 on CCC cells, as described above, were equal to those on known solid cancer cell lines, the effects of apoptosis induction and cell cycle arrest were confirmed to be smaller.

The morphologic changes observed by May-Giemsa staining was a large number of cells containing vacuoles in their cytoplasms in TFK-1 of the VK2-treated group. Since this change was rarely or never observed in the control group, it was considered due to the effects of VK2 (Fig. 5). In addition, the increased number of AVOs observed with acridine orange staining and the increased number of autophagosomes observed by electron microscopy demonstrated that autophagy had been induced in the VK2-treated CCC cells (Fig. 6). Cytoplasmic vacuolation became obvious at $48 \mathrm{~h}$ after the addition of VK2 and accounted for $~ 90 \%$ of the TFK-1 cultured cells at $96 \mathrm{~h}$ (Fig. 7).

Autophagy increases with stimulation by starvation and is a biological phenomenon that occurs as a cytoprotective reaction for cells to survive $(13,14)$. Apoptosis is considered to be type I programmed cell death (PCD), while autophagy is considered to be type II PCD (12). Morphologically, apoptosis involves nuclear changes such as fragmentation and condensation, while autophagy is characterized by few nuclear changes and increased cytoplasmic autophagic vesicles, which is highly consistent with the altered nuclear morphology observed in the VK2-treated TFK-1 (12). This suggested that the antitumor effects of VK2 result from autophagic cell death, not from apoptosis and cell cycle death. There have been reports on autophagy-related cell death in certain types of tumor cell lines including breast cancer cell lines (17) and malignant glioma cell lines $(29,30)$.

It was reported that new alkylating agents, temozolomide (TMZ) and arsenic trioxide $\left(\mathrm{As}_{2} \mathrm{O}_{3}\right)$, induce autophagy in malignant glioma cell lines (30) and that histone deacetylase inhibitors, sodium butyrates and suberoylanilide hydroxamic acid (SAHA), induce autophagy in Bcl-XL overexpressing HeLa cells (31). The addition of etoposide can induce autophagy even in Bax/Bak-double knock-out murine embryonic fibroblasts (MEF), and inhibiting this autophagy pathway by siRNA or 3-methyladenine results in suppressed cytotoxic effects of etoposide, indicating that this autophagy represents autophagic cell death and is an essential process to induce cell death (32). Thus, when autophagy occurs in association with cell death, it is controversial whether autophagy itself causes autophagic cell death or protective autophagy is induced in order to prevent cell death. Also in the present study, non-apoptotic cell death and autophagy in CCC cells induced by VK2 were observed. Whether the autophagy observed in this study is cytoprotective or contributory to cell death induction from a biological aspect remains to be established in future studies.

Although the $\mathrm{IC}_{50}$ for $\mathrm{CCC}$ cells was higher than that for leukemia cells, it was considered to be equal to or lower than that for hepatocellular carcinoma, which is the same type of solid tumor and the chemoprevention effects of VK2 have been confirmed. In addition, because of hepatic metabolism, VK2 has higher tissue concentrations in the liver than in other organs, and after being metabolized by the liver, it is excreted in bile. It has been confirmed that after a single administration of $\mathrm{VK} 2$ to rats, $79.0 \%$ of $\mathrm{VK} 2$ is excreted into bile (33). In dogs administered with ${ }^{14} \mathrm{C}$-menatetrenone, it is known that its radioactive concentration in bile remains extremely high for long periods in comparison to other organs and the maximum biliary level is 51.2 times the blood level $(34,35)$. Although there have been no reports on the investigation of tissue concentrations, since VK2 levels in cholangiocellular carcinoma tissue are expected to be higher than in the tissue of solid tumors other than hepatobiliary tumors, clinical applications of VK2 are anticipated in this disease entity. 


\section{Acknowledgements}

This study was supported by the 'Third-Term Comprehensive Control Research for Cancer' conducted by the Ministry of Health, Labor and Welfare of Japan. We are indebted to Professor J. Patrick Barron of the International Medical Communication Center of Tokyo Medical University, and would like to thank Ms. Ayako Hirota and Ms. Minako Suzuki for their expert technical assistance.

\section{References}

1. Miyazawa K, Yaguchi M, Funato K, Gotoh A, Kawanishi Y, Nishizawa Y, Yuo A and Ohyashiki K: Apoptosis/differentiationinducing effects of vitamin $\mathrm{K} 2$ on HL-60 cells: dichotomous nature of vitamin K2 in leukemia cells. Leukemia 15: 1111$1117,2001$.

2. Yokoyama T, Miyazawa K, Yoshida T and Ohyashiki K: Combination of vitamin $\mathrm{K} 2$ plus imatinib mesylate enhances induction of apoptosis in small cell lung cancer cell lines: Int J Oncol 26: 33-40, 2005.

3. Yoshida T, Miyazawa K, Kasuga I, Yokoyama T, Minemura K, Ustumi K, Aoshima M and Ohyashiki K: Apoptosis induction of vitamin $\mathrm{K} 2$ in lung carcinoma cell lines: the possibility of vitamin K2 therapy for lung cancer. Int J Oncol 23: 627-632, 2003.

4. Tokita H, Tsuchida A, Miyazawa K, Ohyashiki K, Katayanagi S, Sudo H, Enomoto M, Takagi Y and Aoki T: Vitamin K2-induced antitumor effects via cell-cycle arrest and apoptosis in gastric cancer cell lines. Int J Mol Med 17: 235-243, 2006.

5. Bouzahzah B, Nishikawa Y, Simon D and Carr BI: Growth control and gene expression in a new hepatocellular carcinoma cell line, Hep40: inhibitory actions of vitamin K. J Cell Physiol 165: 459-467, 1995.

6. Miyazawa K, Nishimaki J, Ohyashiki K, Enomoto S, Kuriya S, Fukuda R, Hotta T, Teramura M, Mizoguchi H, Uchiyama T and Omine M: Vitamin K2 therapy for myelodysplastic syndromes (MDS) and post-MDS acute myeloid leukemia: information through a questionnaire survey of multi-center pilot studies in Japan. Leukemia 14: 1156-1157, 2000.

7. Yaguchi M, Miyazawa K, Otawa M, Ito Y, Kawanishi Y and Toyama K: Vitamin K2 therapy for a patient with myelodysplastic syndrome. Leukemia 13: 144-145, 1999.

8. Mizuta T, Ozaki I, Eguchi Y, Yasutake T, Kawazoe S, Fujimoto K and Yamamoto $\mathrm{K}$ : The effect of menatetrenone, a vitamin K2 analog, on disease recurrence and survival in patients with hepatocellular carcinoma after curative treatment: a pilot study. Cancer 106: 867-872, 2006.

9. Wu FY, Liao WC and Chang HM: Comparison of antitumor activity of vitamins $\mathrm{K} 1, \mathrm{~K} 2$ and $\mathrm{K} 3$ on human tumor cells by two (MTT and SRB) cell viability assays. Life Sci 52: 1797-1804, 1993.

10. Parekh HK, Mansuri-Torshizi H, Srivastava TS and Chitnis MP: Circumvention of adriamycin resistance: effect of 2-methyl-1, 4-naphthoquinone (vitamin K3) on drug cytotoxicity in sensitive and MDR P388 leukemia cells. Cancer Lett 61: 147-156, 1992.

11. Orimo H, Shiraki M, Tomita A, Morii H, Fujita T and Ohata M: Effects of menatetrenone on the bone and calcium metabolism in osteoporosis: a double-blind placebo-controlled study. J Bone Miner Metab 16: 106-112, 1998.

12. Kondo Y, Kanzawa T, Sawaya R and Kondo S: The role of autophagy in cancer development and response to therapy. Nat Rev Cancer 5: 726-734, 2005

13. Kuma A, Hatano M, Matsui M, Yamamoto A, Nakaya H, Yoshimori T, Ohsumi Y, Tokuhisa T and Mizushima N: The role of autophagy during the early neonatal starvation period. Nature 432: 1032-1036, 2004

14. Tsukada $\mathrm{M}$ and Ohsumi Y: Isolation and characterization of autophagy-defective mutants of Saccharomyces cerevisiae. FEBS Lett 333: 169-174, 1993.

15. Qu X, Yu J, Bhagat G, Furuya N, Hibshoosh H, Troxel A, Rosen J, Eskelinen EL, Mizushima N, Ohsumi Y, Cattoretti G and Levine B: Promotion of tumorigenesis by heterozygous disruption of the beclin 1 autophagy gene. J Clin Invest 112: 1809-1820, 2003.
16. Yue Z, Jin S, Yang C, Levine AJ and Heintz N: Beclin 1, an autophagy gene essential for early embryonic development, is a haploinsufficient tumor suppressor. Proc Natl Acad Sci USA 100: 15077-15082, 2003.

17. Scarlatti F, Bauvy C, Ventruti A, Sala G, Cluzeaud F, Vandewalle A, Ghidoni R and Codogno P: Ceramide-mediated macroautophagy involves inhibition of protein kinase $\mathrm{B}$ and up-regulation of beclin 1. J Biol Chem 279: 18384-18391, 2004.

18. Yu L, Alva A, Su H, Dutt P, Freundt E, Welsh S, Baehrecke EH and Lenardo MJ: Regulation of an ATG7-beclin 1 program of autophagic cell death by caspase-8. Science 304: 1500-1502, 2004.

19. Yaguchi M, Miyazawa K, Otawa M, Katagiri T, Nishimaki J, Uchida Y, Iwase O, Gotoh A, Kawanishi Y and Toyama K: Vitamin K2 selectively induces apoptosis of blastic cells in myelodysplastic syndrome: flow cytometric detection of apoptotic cells using APO2.7 monoclonal antibody. Leukemia 12: 1392-1397, 1998 .

20. Tsuchida A, Nagae I, Saitoh H, Ikeda T, Tanabe Y, Takahashi S, Aoki T and Itoi T: Clin Gastroenterol 21: 705-709, 2006.

21. Saijyo S, Kudo T, Suzuki M, Katayose Y, Shinoda M, Muto T, Fukuhara K, Suzuki T and Matsuno S: Establishment of a new extrahepatic bile duct carcinoma cell line, TFK-1. Tohoku J Exp Med 177: 61-71, 1995.

22. Yoshida K, Tomizawa H, Ota T, Nagashima T, Kikuchi H, Watanabe H, Hashizaki $\mathrm{K}$ and Yonaha A: Establishment and characterization of human cholaginocarcinoma, MEC, producing carbohydrate antigen 19-9. Hum Cell 3: 346-351, 1990.

23. Miyagiwa M, Ichida T, Tokiwa T, Sato J and Sasaki H: A new human cholangiocellular carcinoma cell line (HuCC-T1) producing carbohydrate antigen 19-9 in serum-free medium. In Vitro Cell Dev Biol 25: 503-510, 1989.

24. Paglin S, Hollister T, Delohery T, Hackett N, McMahill M, Sphicas E, Domingo D and Yahalom J: A novel response of cancer cells to radiation involves autophagy and formation of acidic vesicles. Cancer Res 61: 439-444, 2001.

25. Traganos F and Darzynkiewicz Z: Lysosomal proton pump activity: supravital cell staining with acridine orange differentiates leukocyte subpopulations. Methods Cell Biol 41: 185-194, 1994.

26. Stankiewicz M, Jonas W, Hadas E, Cabaj W and Douch PG: Supravital staining of eosinophils. Int J Parasitol 26: 445-446, 1996.

27. Nishimaki J, Miyazawa K, Yaguchi M, Katagiri T, Kawanishi Y, Toyama K, Ohyashiki K, Hashimoto S, Nakaya K and Takiguchi T: Vitamin K2 induces apoptosis of novel cell line established from a patient with myelodysplastic syndrome in blastic transformation. Leukemia 13: 1399-1405, 1999.

28. Wang Z, Wang M, Finn F and Carr BI: The growth inhibitory effects of vitamins $\mathrm{K}$ and their actions on gene expression. Hepatology 22: 876-882, 1995.

29. Kanzawa T, Kondo Y, Ito H, Kondo S and Germano I: Induction of autophagic cell death in malignant glioma cells by arsenic trioxide. Cancer Res 63: 2103-2108, 2003.

30. Kanzawa T, Germano IM, Komata T, Ito H, Kondo Y and Kondo S: Role of autophagy in temozolomide-induced cytotoxicity for malignant glioma cells. Cell Death Differ 11: 448457, 2004.

31. Shao Y, Gao Z, Marks PA and Jiang X: Apoptotic and autophagic cell death induced by histone deacetylase inhibitors. Proc Natl Acad Sci USA 101: 18030-18035, 2004.

32. Shimizu S, Kanaseki T, Mizushima N, Arakawa-Kobayashi S, Mizuta T, Thompson CB and Tsujimoto Y: Role of Bcl-2 family proteins in a non-apoptotic programmed cell death dependent on autophagy genes. Nat Cell Biol 6: 1221-1228, 2004.

33. Sano Y, Tadano K, Kaneko K, Kikuchi K and Yuzuriha T: Metabolic fate of menatetrenone in rats - absorption, distribution, metabolism and excretion after a single oral administration. Jpn Pharmacol Ther 23: 2659-2667, 1995.

34. Sano Y, Tadano K, Kikuchi K, Kaneko K, Yuzuriha T, Kimura T and Murayama Y: Metabolic fate of menaquinone-4 in dogs (I): Absorption, distribution, metabolism and excretion after a single oral administration. Drug Metab Pharmacokinet 12: 48-57, 1997.

35. Sano Y, Tadano K, Kikuchi K, Kaneko K, Yuzuriha T, Kimura T and Murayama Y: Metabolic fate of menaquinone-4 in dogs (II): Absorption, distribution, metabolism and excretion after a single oral administration. Drug Metab Pharmacokinet 12: 58-67, 1997. 